\title{
Molecular tissue typing in renal transplantation: Initial experience from a tertiary care Naval Hospital
}

\author{
Mahendra N. Mishra, Haresh Mani*, Vinod K. Saxena**, Mahendra K. Gupta*** \\ Dept. of Pathology, 166 Military Hospital, C/O 56 APO, *Dept. of Pathology, Armed Forces Medical College, Pune - 411 040, **Dept. of \\ Pathology, INHS Asvini, Colaba, Mumbai - 400 005, ${ }^{* \star *}$ Dept. of Pathology, Bombay Hospital, New Marine Lines, Mumbai, India
}

BACKGROUND: During the last five years the proportion of living unrelated kidney transplants has increased and DNA tissue-typing methods have become popular in India. This study was carried out to compare the results of tissue - typing by serology and sequence specific primers (SSP) and study the usefulness of 'episode' allograft biopsies for diagnosis of acute graft dysfunction.

MATERIALS AND METHODS: DNA was extracted from whole blood using Qiagen kit. Samples from 60 individuals including thirty patients and their donors were typed by serology and SSP. Fifteen allograft biopsies were performed for suspected acute rejection (AR) cases.

RESULTS: Both alleles of HLA - A, B and DR antigen could be determined in 86,65 and $90 \%$ of samples by SSP respectively. There was a discrepancy of $16-40 \%$ between SSP and serology. Acute rejection was confirmed in 8/15 biopsies. Graft survival rates were 83 and $76 \%$ at one and two years respectively. Neither the graft survival nor the number of AR episodes showed any correlation with the extent of HLA mismatch. SSP was useful in defining $A^{*} 68$, $A^{*} 66, A^{*} 69$ and $A^{*} 33$ alleles at private level and $A^{*} 36, A^{*} 74$ and $A^{*} 03$ alleles which were blank on serology.

CONCLUSIONS: SSP has become popular in India due to its simplicity, superior results especially for class II HLA alleles: and episode allograft biopsy is adequate for followup of kidney recipients.

Key words: Allograft biopsy, graft rejection, tissue-typing

\section{Introduction}

Kidney transplantation is the optimal treatment for chronic renal failure. The predominant clinical application of HLA typing is in solid organ and bone marrow transplantation (BMT). HLA antigens of A, B (Class I) and DR (Class II) loci are particularly relevant in renal transplantation. Patients with a greater degree of match have been observed to have fewer acute rejection (AR) episodes ${ }^{[1]}$ and also better graft survival. ${ }^{[2-4]}$ The degree of HLA matching is relevant for cadaver kidney allocation and in living donor selection if choice for donors with better match is available. In India however, due to paucity of cadaver kidneys, the choice of donors is rather limited. Most tissue-typing laboratories commence HLA typing by serology and then gradually adopt molecular methods - first for class II and subsequently for class I. In renal transplantation, low or medium-resolution tissue-typing is adequate, whereas in unrelated BMT it is always followed with high-resolution typing.

\section{Materials and Methods}

This study was done at a government hospital during the period August 2002 to September 2004. All potential donors $(n=30)$ were subjected to a thorough medical check-up after which approval from Directorate of Medical and Scientific Research; Maharashtra Government was obtained for all transplantations. Clearance of the Institute's ethical committee was also obtained. Donor specific T cell cross match was done twice by $\mathrm{NIH}$ two stage microlymhocytoxicity assay ${ }^{[5]}$ once at the time of HLA typing and was repeated $48 \mathrm{~h}$ before transplantation. HLA typing of 60 samples (patients and donors) was done by serology and SSP. All patients $(n=30)$ were followed-up for two years and allograft biopsy was done in 15 recipients who presented with acute impairment of renal function.

$B$ cell cross match and PRA were done only for five retransplant patients. B cells were separated using immunomagnetic HLA Class II Dynabeads ${ }^{\circledR}$ (Dynal, Norway). Staining of the cells was done using acridine orange/ ethidium bromide. Assessment of viable (green) 
and dead cells (red) was made using a fluorescence microscope (Carl Zeiss, Germany) having a 450/490 nm excitation filter. PRA estimation was done by ELISA using (GTI, USA) kits.

DNA Extraction was done from whole blood collected in EDTA vacutainers using QIA $^{\circledR}$ DNA Mini Kit (Qiagen, Germany) by adsorption of DNA onto silica-gel membrane followed by its elution as described by Knipper et al. ${ }^{[6]}$ DNA yield and purity was determined by standard methods after which it was stored at $-30^{\circ} \mathrm{C}$ until amplification by SSP. Tissue-typing by SSP was done using kits from (Biotest AG. Dreiech, Germany) for 40 samples; and for 20 samples using kits from (GenoVision, USA). The procedure given in product insert for preparation of master - mix and for amplification of alleles was followed as described in a previous study. ${ }^{[7]}$ The amplified products were visualized on $2 \%$ agarose gel in $0.5 \times$ TBE buffer. Evaluation and analysis for 20 samples amplified using GenoVision, (USA) SSP kits was done using Helmberg "Virtual sequencing" software while for the remaining samples amplified by Biotest kits, analysis was done manually using data sheets.

\section{Immunosupression}

Patients received standard conventional triple-drug immunosuppression using cyclosporine, azathioprine and prednisolone. Rejection episodes were treated with intravenous methyl prednisolone $(15 \mathrm{mg} / \mathrm{kg}$ body weight), subject to a maximum of one gram daily on three consecutive days. Steroid resistant rejection was treated with either OKT-3 or Anti-thymocyteglobulin.

Post transplant follow-up was done based on clinical evaluation, urine output, blood urea and creatinine levels. In suspected cases of AR diethylene pentamino acetic acid scan was followed by allograft biopsy. Allograft biopsy was performed not as per any protocol but when there was clinical or biochemical evidence of graft dysfunction ("episode biopsy"). Fifteen renal biopsies were performed under ultrasound guidance using Monopty Semiautomatic Biopsy Gun with cutting edge (Bard Inc). Histological diagnosis was made according to the Banff working classification. ${ }^{[8]}$ Borderline function loss implied serum creatinine $>2.5$ $\mathrm{mg} / \mathrm{dl}$. Graft survival was defined as the number of months of graft functioning until either graft loss or death of the patient. Graft loss was defined as return to dialysis or serum creatinine more than $6 \mathrm{mg} / 100$ $\mathrm{ml}$.

Statistical analysis: Of the accrued data was done by the Chi-square test and by the Kaplan - Meier survival curve. The patients were categorized into two groups for statistical analysis: those with $<3$ antigen mismatch and those with four to six antigen mismatches.

\section{Results}

\section{Patient and donor data}

The cause of ESRD in 23 patients was chronic glomerulonephritis (CGN); focal segmental glomerulosclerosis, membranoproliferative glomerulonephritis, chronic interstitial nephropathy, adult polycystic kidney disease, diabetic nephropathy, Immunoglobulin A nephropathy and systemic lupus nephritis - in one case each. Twenty-six patients $(87.5 \%)$ who received a renal allograft were males and remaining four $(12.5 \%)$ were females. Twenty-five allografts were first grafts (83\%) and five were regrafts. There was a slight female preponderance among donors $(63 \%)$. The mean age of the recipients and donors was 32.7 and 41.8 years respectively. Donors were categorized live related donors $\operatorname{LRD}(n=13)$, which included parents and siblings; and LURD $(n=17)$, which included all non first-degree relatives. The donorrecipient relationship for 30 kidney recipients was as follows: parent - 9, wife - 7 , sibling - 4 and other non first-degree relatives-10.

\section{Cross match results}

Five patients had a donor specific initial positive initial cross match result including three $T$ cell cross match positive and two with $\mathrm{B}$ cell cross match positive results. However subsequently four patients had a final negative donor specific cross match result and the solitary patient who tested positive could not get a donor for third allograft during the period of this study.

\section{DNA quality and quantity}

The quantity of DNA obtained by column-based method from $200 \mu$ of whole blood varied between $2.5-6 \mu \mathrm{g}$. The ratio of absorbance at $260 \mathrm{~nm}$ to absorbance at $280 \mathrm{~nm}\left(\mathrm{~A}_{260} / \mathrm{A}_{280}\right)$ was 1.5-1.8 for all DNA samples. 


\section{Comparison of serology and SSP}

Results for 60 samples is shown in Table 1 . In 36 samples (60\%) all six alleles of A, B and DR antigen were determined by SSP. Both alleles of A, B and DR antigen were identified in $87.5,65$ and $91 \%$ of samples respectively by SSP. The most frequent class I alleles were $A^{*} 24^{[9]}, A^{*} 02 ; B^{*} 51, B^{*} 35^{[9]}$. The most common class II antigens were DRB $1^{*} 15$ and $1{ }^{*} 07$. Genomic DNA from eight samples is seen in Figure 1 shows the gel analysis of HLA typing results of one patient. Using SSP we were able to define both antigens of HLA $A$ in $3 / 6$ samples which were blank on serology and we also identified 3 splits $A^{*} 74, A^{*} 69$ and $A^{*} 66$ at private level, that had been identified at public level by serology. The results of HLA-B serotyping were similar to DNA typing while the results of DNA based DR typing was far superior to serology [Table 1]. Both DR alleles were determined in 27/30 samples by SSP as compared to only $20 / 30$ samples by serology. A discrepancy of $16-40 \%$ was seen between the results of serology and DNA typing including missed alleles,

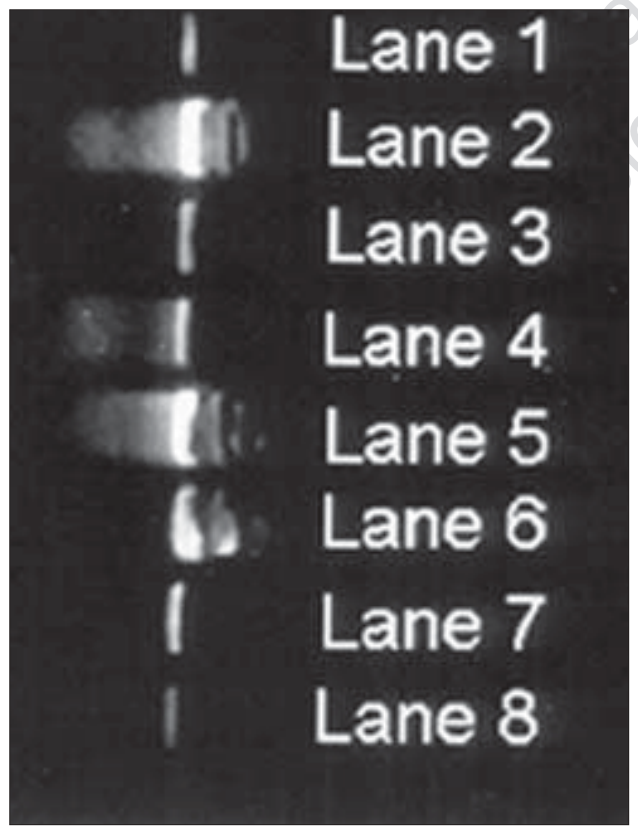

Figure 1: Genomic DNA of 8 samples are seen as a crisp line close to origin of the well in lanes 1-8. unidentified alleles at split-level.

HLA Mismatches: There were no patients with 0 or 1 mismatch (MM). The number of mismatched alleles for patients was as follows: 2 allele MM $(n=1), 3$ alleles MM $(n=16), 4$ alleles MM $(n=7), 5$ allele MM $(n=4), 6$ allele MM ( $\mathrm{n}=2)$.

Acute Rejection: Was suspected in 15 cases, but was confirmed in 8 patients after allograft biopsy of which six recipients were mismatched for 3 alleles, while two had $>3$ allele MM. With antirejection treatment the AR was completely reversed in five patients and partly reversed in three recipients. One-third time recipient with historical positive donor specific BCXM developed irreversible hyper acute rejection. Allograft biopsy was adequate for the diagnosis of causes of graft dysfunction except for in four patients of which two showed a normal histology, one was inadequate and had to be repeated, while the last showed borderline changes. Allograft biopsy was useful for discerning other causes of impaired renal function: Cyclosporine toxicity, acute tubular necrosis and interstitial fibrosis.

Follow up: Twenty-five kidney recipients (83\%) including $4 / 5$ re-transplant patients had a functional graft at the end of one year. Four patients were back on maintenance hemodialysis of which one succumbed to septicemia. At the time of writing this paper remaining three kidney recipients are awaiting retransplant, including the second time recipient who developed hyper acute rejection and had positive donor specific B cell cross match. One recipient died of central nervous system lymphoma with a functional graft within nine months of transplant. Three of the patients with graft loss were mismatched for 3 alleles and two for more than 3 alleles. Neither the number of AR episodes nor the one year graft survival was significantly different in the patients with $<3$ allele $\mathrm{MM}$ and with $>3 \mathrm{MM}$ : $P$ value $>0.05$. Two patients developed insidious loss of allograft function and were on dialysis during the second year of follow-up.

Table 1: Comparison of HLA typing by serology with molecular typing

\begin{tabular}{lcccc}
\hline Antigen & One alleles identified & Both alleles identified & Private alleles identified & Serology - SSP \\
\hline A & & & by SSP & disparity (\%) \\
B & $14(\mathrm{~s}), 8(\mathrm{~m})$ & $46(\mathrm{~s}), 52(\mathrm{~m})$ & 6 & 16 \\
DR & $22(\mathrm{~s}), 21(\mathrm{~m})$ & $38(\mathrm{~s}), 39(\mathrm{~m})$ & 2 & 40 \\
\hline
\end{tabular}

S - Results of samples typed by serology; M - Results of samples typed by SSP, SSP - Sequence specific primers 


\section{Discussion}

An increase in biologically unrelated kidney donors has been observed in India ${ }^{[1,7]}$ which necessitates a greater requirement for correct allele assignment. The basis of cadaver kidney allocation in Eurotransplant and United Network for Organ Sharing is HLA matching between recipient and donor but the situation is different in our country. Accurate allele assignment is useful in India not for kidney allocation or for living donor selection, but for prognostication because cadaver kidneys and living donors are scarce. Shortcomings of serological typing specially with respect to DR typing have been shown and in laboratories with workload of up to two hundred samples the cost of SSP and serology has been found to be similar. ${ }^{[7]}$ In this study the SSP typing results for DR and $A$ antigens were satisfactory, but both alleles of HLA B could not be determined in $65 \%$ of samples. Amplification failures were observed with HLA-B typing and many reactions did not show both the control and positive band. The $B$ locus is most polymorphic and it is possible that with more experience we would have got better results. Due to our limited budget it was not possible to repeat SSP typing on same sample and with further experience it will be possible to improve upon HLA B typing results.

SSP was useful in defining both alleles of HLA A antigen at private level in six patients blank HLA-A alleles in serology which were identified by SSP included: $A^{*} 36$ in two related samples and $A^{\star} 74, A^{\star} 03$ in one sample each [Figure 2]. In 6/8 samples both alleles of DR antigen which were not identified on serology, they could be detected by SSP and the results of SSP were differed from serology for five patients. With SSP we were able to also identify DR antigens at private level in four samples [Table 1], which were identified at public level by serology.

Many other workers have reported disparity between serology and DNA tissue-typing. ${ }^{[9-13]}$ This has ranged from $9 \%$ for HLA - $\mathrm{A}^{[9]}$ to $83 \%$ for DR antigens. ${ }^{[13]}$ This implies that DNA tissue-typing is imperative for better results, particularly for BMT where error rate must not exceed $2 \%$ for HLA - A-B and $1.5 \%$ for HLA DRB typing. ${ }^{[14]}$

HLA matching for DPB1, ${ }^{[15]}$ HLA $C$ and DQ matching ${ }^{[16]}$ have been shown to be associated with

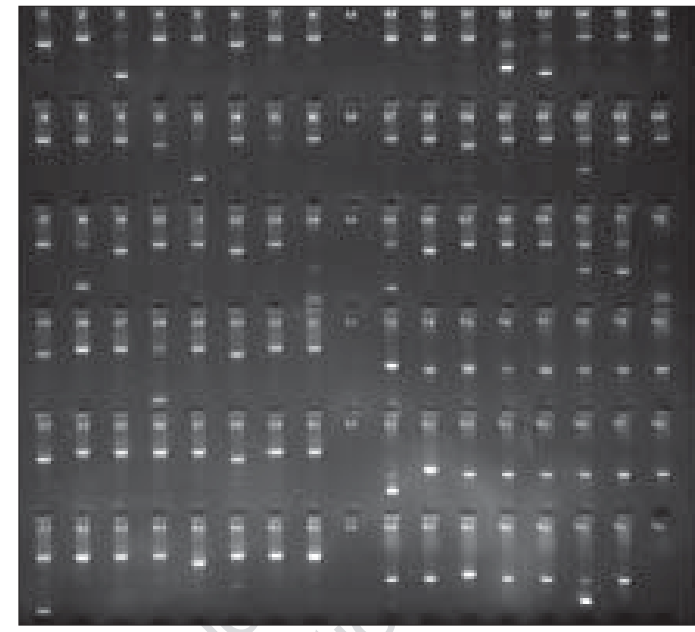

Figure 2: SSP ABDR typing results of one sample showing 1069 base pair control bands closer to point of application and amplified bands, being of smaller, size and move further away. Results: Positive bands: 3, 13, $18,28,41,52,53,62,65,70,71,81$, and 94 . Using Helmberg score and software based interpretation the alleles identified were: $A^{\star} 0308, A^{\star} 32$; $B^{\star} 4410, B^{\star} 5612$, w4; DRB1*07, DRB1*07, B4.

better graft survival but this becomes more expensive and is not required in India routinely for living kidney donors. Opelz et a ${ }^{[17]}$ had shown the beneficial effect of HLA matching on graft survival in 1,50,000 kidney recipients which also included 3271 Asians from multiple centers that participated in collaborative transplant study (CTS). In this study neither the graft survival nor the AR episodes showed a correlation with HLA MM. However, this is too small a study to be extrapolated. Bryan et $a^{{ }^{[18]}}$ in their study also reported no correlation between HLA matching and graft survival. Foss et $a^{[19]}$ in a study on 141 LURD found higher frequency of AR episodes in HLA mismatched groups, but in this study allograft survival was not affected by HLA mismatch. In today's era of excellent immunosuppressants there is little difference between graft outcomes of those with one and six mismatch and no patient should be denied a kidney for want of HLA mismatch.

Histopathological examination of 15 kidney biopsies from suspected cases of AR was useful for establishing the diagnosis in all but one patient and in our opinion protocol biopsy is not imperative for routine follow-up of kidney recipients. In conclusion because of its simplicity, comparable cost and more precise results we advocate use of SSP typing and "episode" graft biopsies for the management and followup of kidney recipients in India. 


\section{Acknowledgments}

The authors are grateful to DGAFMS for sanctioning the armed forces research project for the above study and to Dr. Mrs. Vrinda Trikkinad for allowing use of laboratory facilities.

\section{References}

1. Panigrahi A, Agarwal SK, Kanga U, Guleria S, Bhowmik D, Dash SC, et al. Influence of HLA compatibility on renal graft survival using live unrelated and cadaver donors in India. Indian J Med Res 2002;115:158-64.

2. Leivstad T, Reisaeter AV, Brekke IB, Vartdal F, Thorsby E. The role of HLA matching in renal transplantation: Experience from one center. Rev Immunogenet 1999;1:343-50.

3. Terasaki PI. The HLA-matching effect in different cohorts of kidney transplant recipients. Clin Transplant 2000;14:497-514.

4. Bresnashan BA, Johnson CP, McIntosh MJ, Stableein D, Hariharan S. A comparison between recipients receiving matched kidney and those receiving mismatched kidneys from the same cadaver donor. Am J Transplant 2002;2:366-72.

5. Terasaki PI, Mc Clellend JD. Microdroplet assay of human serum cytotoxins. Nature 1964;204:998-1000.

6. Knipper AJ, Hakenberg P, Enczmann J, Kuhrober A, Kiesel U, Kogler G, et al. HLA-DRB1,3,4,5 and -DQB1 allele frequencies and HLA- DR /DQ linkage disequilibrium of 231 German caucasoid patients and their corresponding 821 potential unrelated stem cell transplants. Hum Immunol 2000;61:605-14.

7. Mishra MN, Mani H, Narula AS, Saxena VK. HLA TypingA comparison of serology and DNA techniques. Int J Hum Genet 2004;4:151-3.

8. Racusen LC, Soiez K, Colvin RB, Bonsib SM, Castro MC, Cavallo $\mathrm{T}$, et al. The Banff 97 working classification of renal allograft pathology. Kidney Int 1999;55:713-23.

9. Kulcsarova E, Kralovicova J, Parnicka Z, Ferencik S, Buc $M$. Comparison of the results of HLA typing using serologic and molecular genetics methods. Bratisl Lek Listy
2000;101:134-7.

10. Woszczek G, Borowiec M, Mis M, Gorska M, Kowalski ML. Comparison of serological and molecular (PCR-SSP) techniques off HLA-DR typing in clinical laboratory routine. Ann Transplant 1997;2:39-42.

11. Poli F, Scalamogna M, Crepiatico L, Macchi B, Misto R, Nocco A, et al. Comparison of serological and molecular typing for HLA-A and B on cord blood lymphocytes. Tissue Antigens 1998;51:67-71.

12. Laurenti L, Perrone MP, Bafti MS, Ferari F, Screnci M, Pasqua I, et al. HLA typing strategies in a cord blood bank. Haematologica 2002;87:851-4.

13. Mytilineos J, Lempert M, Scherer S, Schwarz V, Opelz G. Comparison of DNA PCR- SSP typing results for HLA- A and HLA- B in 421 black individuals: A Collaborative Transplant Study report. Hum Immunol 1998;59:512-7.

14. Hurley CK, Wade JA, Oudshoorn M, Middleeton D, Kukuruga D, Navarrete C, et al. A special report: Histocompatibility testing guidelines for hematopoietic stem cell volunteer donors. Tissue Antigens 1999;53:394-406.

15. Opelz G, Wujaciak T, Dohler B, Scherer S, Mytilineos J. HLA compatibility and organ transplant survival Collaborative Transplant Study. Rev Immunogenet 1999;1:334-42.

16. Rees MT, Darke C. HLA-A, B, C, DRB1, DQB1 matching heterogeneity in 'favourably matched' kidney recipients. Transpl Immunol 2003;12:73-8.

17. Opelz G. HLA matching in Asian recipients of kidney grafts from unrelated living or cadaveric donors. The Collaborative Transplant Study. Hum Immunol 2000;61:115-9.

18. Bryan CF, Harrell KM, Mitchell SI, Warady BA, Aeder MI, Luger AM, et al. HLA points assigned in cadaveric kidney allocation should be revisited: An analysis off class II molecularly typed patients and donors. Am J Transplant 2003;3:459-64.

19. Foss A, Leivestad T, Brekke IB, Fauchald P, Bentdal O, Lien B, et al. Unrelated living donors in 141 kidney transplantations. Transplantation 1998;66:49-52.

Source of Support: Nil, Conflict of Interest: None declared. 